\title{
Determination of Rheological Parameters from Measurements on a Viscometer with Coaxial Cylinders
}

\section{F. Rieger}

The paper deals with measurements of non-Newtonian fluids on a viscometer with coaxial cylinders. The procedure for determining the rheological model parameters is recommended for power-law fluids and Bingham plastics.

Keywords: viscometer with coaxial cylinders, power-law fluids, Bingham plastics.

\section{Introduction}

A rotational viscometer with coaxial cylinders is widely used in rheological measurements. Its common configuration consists of an inner rotating cylinder with radius $R_{1}$ and length $L$ and an outer stationary cylinder with radius $R_{2}$ - see Fig. 1. The dependence of shear stress $\tau$ on the Newtonian shear rate $\dot{\gamma}_{N}$ at the specified radius is usually obtained from measurements. The power law and Bingham model are the simplest rheological models. The aim of this paper is to show a way to calculate the parameters of these models from experimental values $\tau$ and $\dot{\gamma}_{N}$. For this purpose, the flow in the viscometer must be analysed.

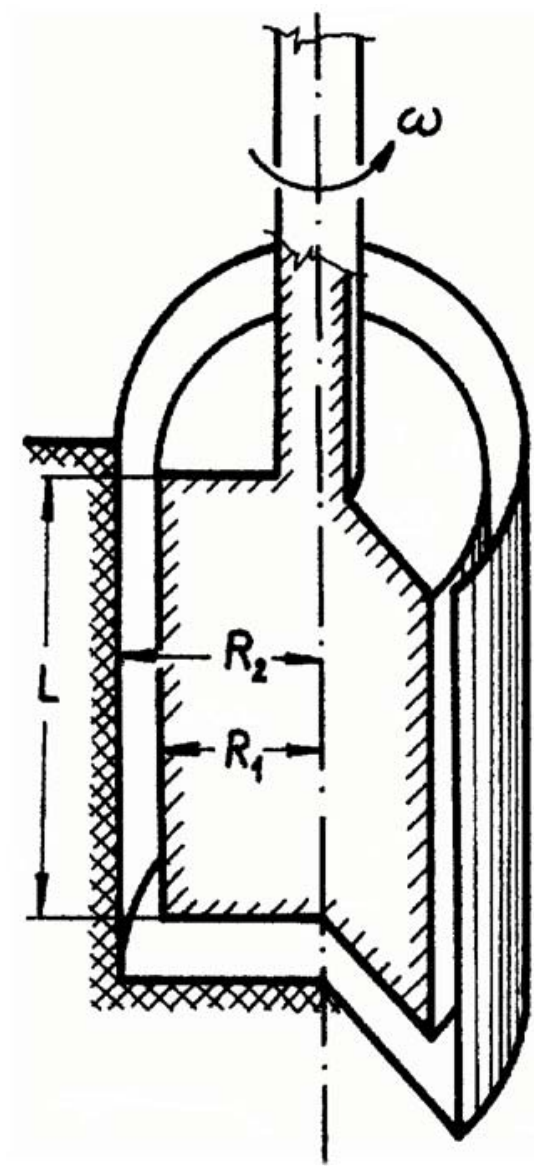

Fig. 1: Viscometer with coaxial cylinders

\section{Theory}

If the influence of the bottom and the interface is neglected, the only non-zero velocity component $u_{\varphi}$ depends in cylindrical coordinates on the radius $r$ only. The component of Cauchy's equation of motion for this case takes the form (see e.g. [1])

$$
\frac{\mathrm{d}}{\mathrm{d} r}\left(r^{2} \tau_{r \varphi}\right)=0
$$

Integrating this equation, we obtain the following relation for shear stress

$$
\tau_{r \varphi}=\frac{C_{1}}{r^{2}}
$$

The shear rate for this type of flow is given by the relation (see e.g. [1])

$$
\dot{\gamma}=r \frac{\mathrm{d}}{\mathrm{d} r}\left(\frac{u_{\varphi}}{r}\right) .
$$

\subsection{Power law fluids}

The power law is the simplest model that is widely used for describing the rheological behavior of non-Newtonian fluids. Using this model, the dependence of shear stress on shear rate can be expressed by the following relation

$$
\tau=K|\dot{\gamma}|^{n-1} \dot{\gamma},
$$

where $K$ is the coefficient of consistency and $n$ stands for flow behavior index.

Inserting (2) and (3) into Eq. (4) and taking into consideration that the shear rate in the gap is negative, we obtain

$$
K\left[-r \frac{\mathrm{d}}{\mathrm{d} r}\left(\frac{u_{\varphi}}{r}\right)\right]^{n}=-\frac{C_{1}}{r^{2}} .
$$

Integrating the above equation we obtain

$$
\frac{u_{\varphi}}{r}=\left(-\frac{C_{1}}{K}\right)^{\frac{1}{n}} \frac{n}{2} r^{-\frac{2}{n}}+C_{2} .
$$

For determining integration constants, the following boundary conditions are necessary

$$
\begin{aligned}
& r=R_{1}, \frac{u_{\varphi}}{r}=\omega, \\
& r=R_{2}, \frac{u_{\varphi}}{r}=0 .
\end{aligned}
$$


Using them, the following expression for integration constant $C_{1}$ is obtained

$$
C_{1}=-K\left[\frac{2 \omega}{n\left(R_{1}^{-2 / n}-R_{2}^{-2 / n}\right)}\right]^{n} .
$$

Inserting (8) into (5) the following equation for shear rate can be obtained after rearrangement

$$
\dot{\gamma}=-\frac{2 \omega}{n\left(1-\kappa^{2 / n}\right)}\left(\frac{R_{1}}{r}\right)^{2 / n},
$$

where $\kappa=R_{1} / R_{2}$.

The dependence of the dimensionless shear rate $\dot{\gamma}^{*}=-\dot{\gamma} / \omega$ on the dimensionless coordinate defined by the relation $y^{*}=y /\left(R_{2}-R_{1}\right)$ (where $y$ is the radial distance from the rotating cylinder) for ratio $\kappa=0.5$ and several $n$ values is shown in Fig. 2. The same dependence for ratio $\kappa=0.9$ is shown in Fig. 3. From Figs. 2 and 3 it is obvious that the maximum shear rate is at the inner cylinder $\left(y^{*}=0\right)$ and the minimum is at the outer cylinder $\left(y^{*}=1\right)$. From the above mentioned figures for both $\kappa$ values it can also be seen that the

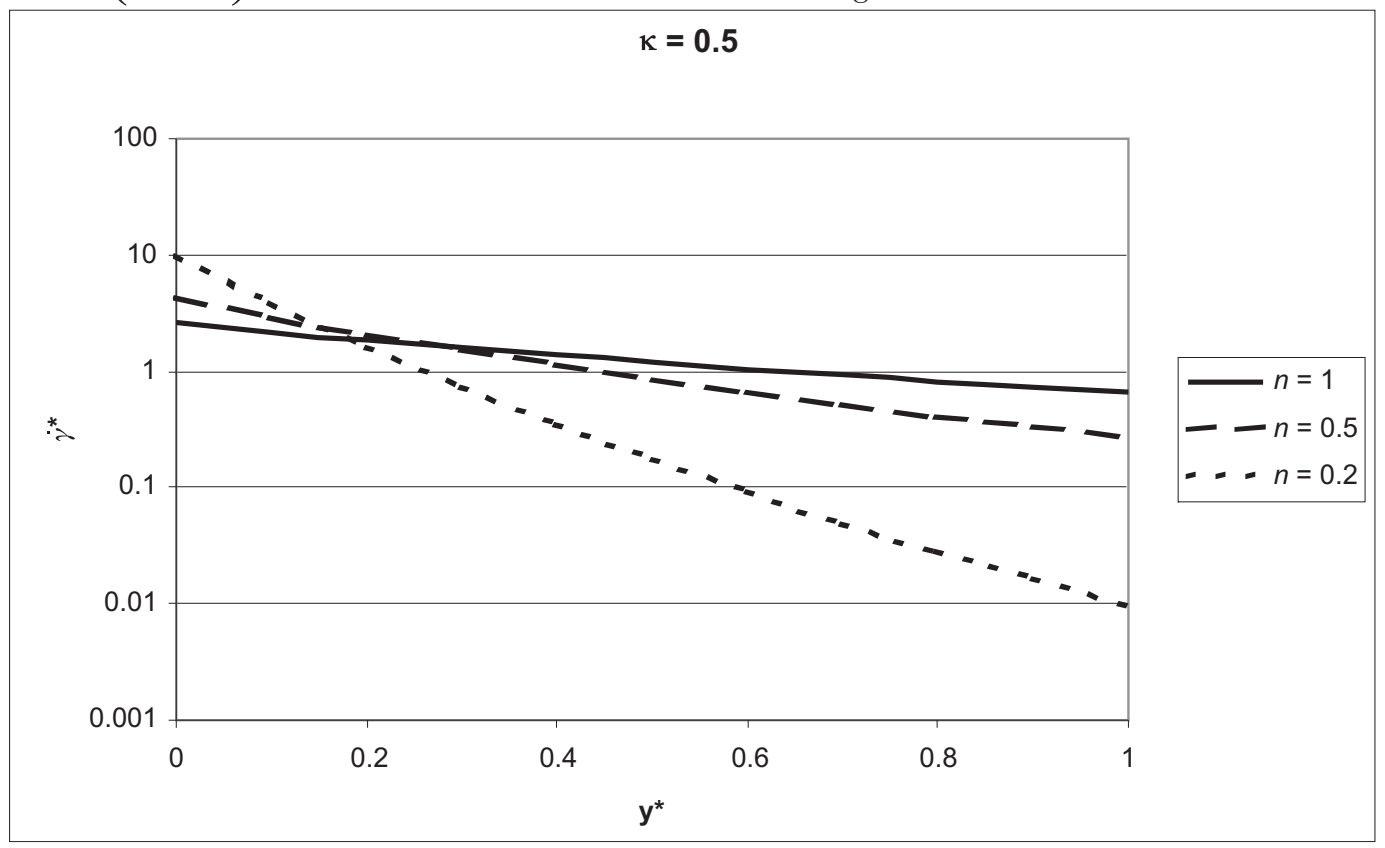

Fig. 2: Dependence of dimensionless shear rate values $\dot{\gamma}^{*}$ on dimensionless distance $y^{*}$ for $\kappa=0.5$ and selected flow behavior index values $n$

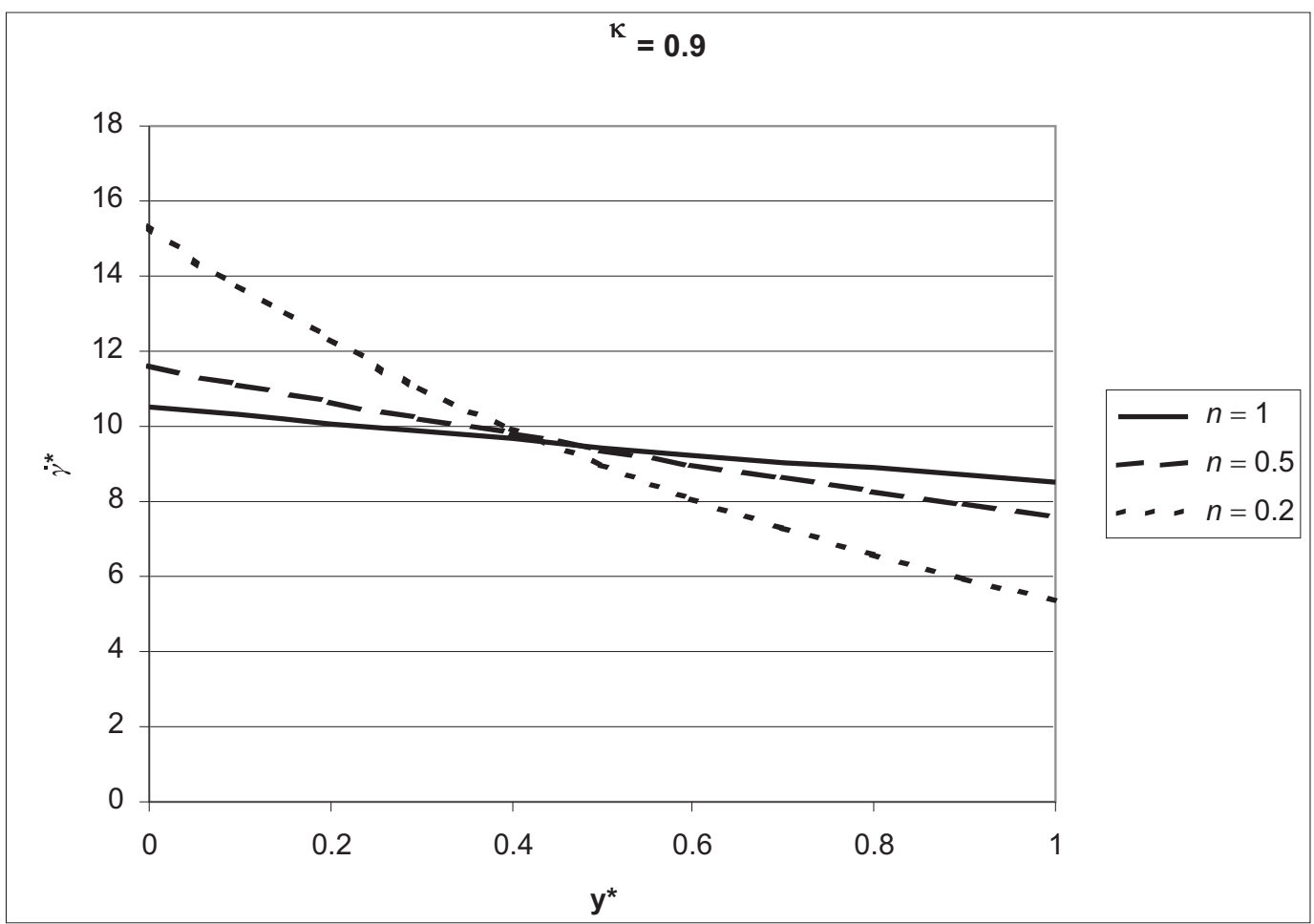

Fig. 3: Dependence of dimensionless shear rate values $\dot{\gamma}^{*}$ on dimensionless distance $y^{*}$ for $\kappa=0.9$ and selected flow behavior index values $n$ 
effect of flow behavior index $n$ on the shape of the shear rate profiles is more pronounced at smaller $\kappa$ value. At $\kappa \rightarrow 1$ (parallel plate asymptote) the flow behavior index $n$ has no effect on the shear rate profiles - the shear rate is constant.

\subsection{Bingham plastics}

The simplest model for viscoplastic behavior is the Bingham model

$$
\dot{\gamma}=0 \text { for }|\tau| \leq \tau_{0}
$$

$$
\tau=\left(\mu_{\mathrm{p}}+\frac{\tau_{0}}{|\dot{\gamma}|}\right) \dot{\gamma} \text { for }|\tau| \geq \tau_{0},
$$

where $\mu_{\mathrm{p}}$ is plastic viscosity and $\tau_{0}$ stands for yield stress.

Inserting (2) and (3) into equation (10b) and taking into consideration that the shear rate in the gap is negative, we obtain

$$
\mu_{\mathrm{p}} r \frac{\mathrm{d}}{\mathrm{d} r}\left(\frac{u_{\varphi}}{r}\right)-\tau_{0}=\frac{C_{1}}{r^{2}}
$$

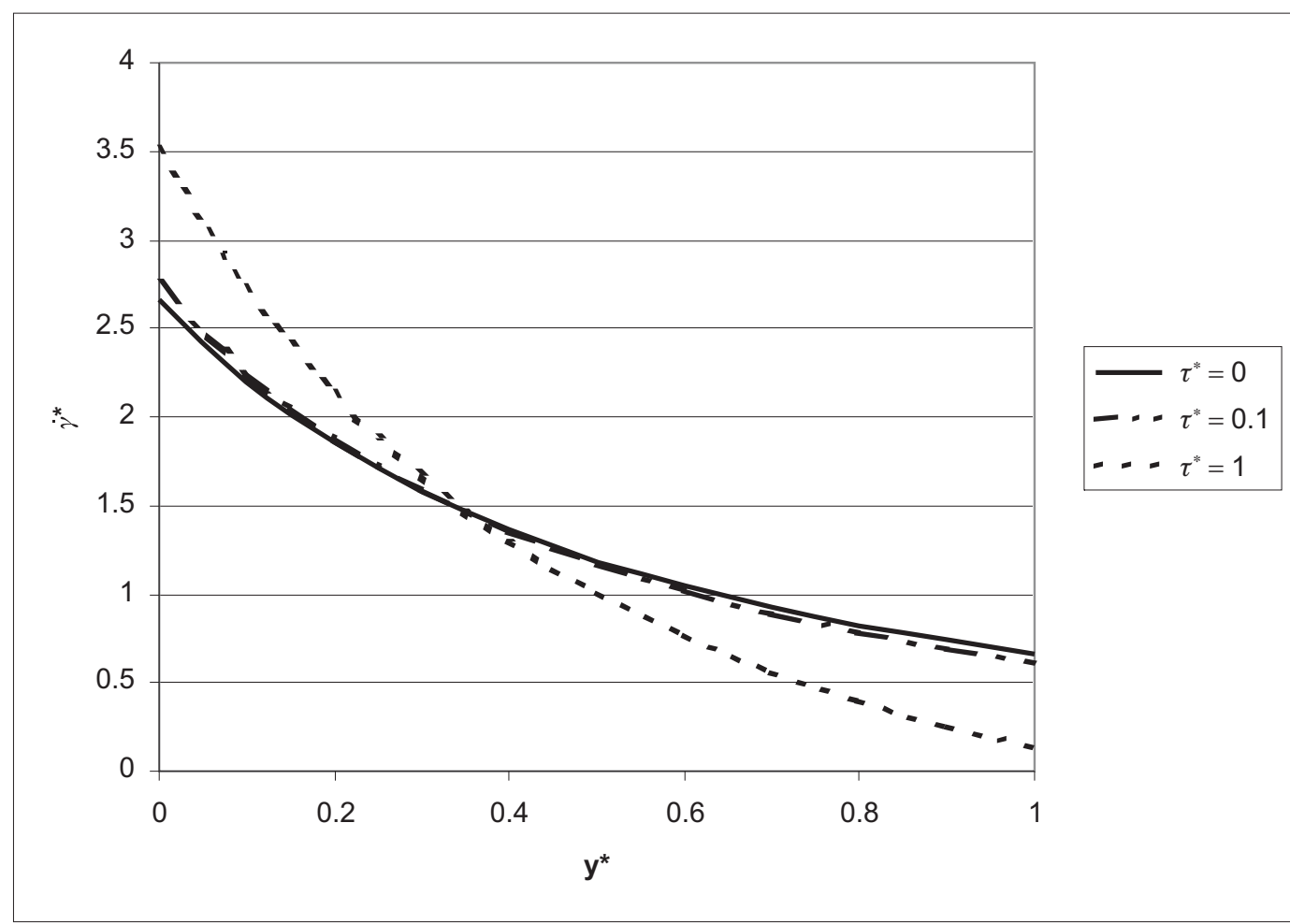

Fig. 4: Dependence $\dot{\gamma}^{*}$ on $y^{*}$ for $\kappa=0.5$ and selected values $\tau^{*}\left(\tau_{\mathrm{c}}^{*}=1.24\right)$

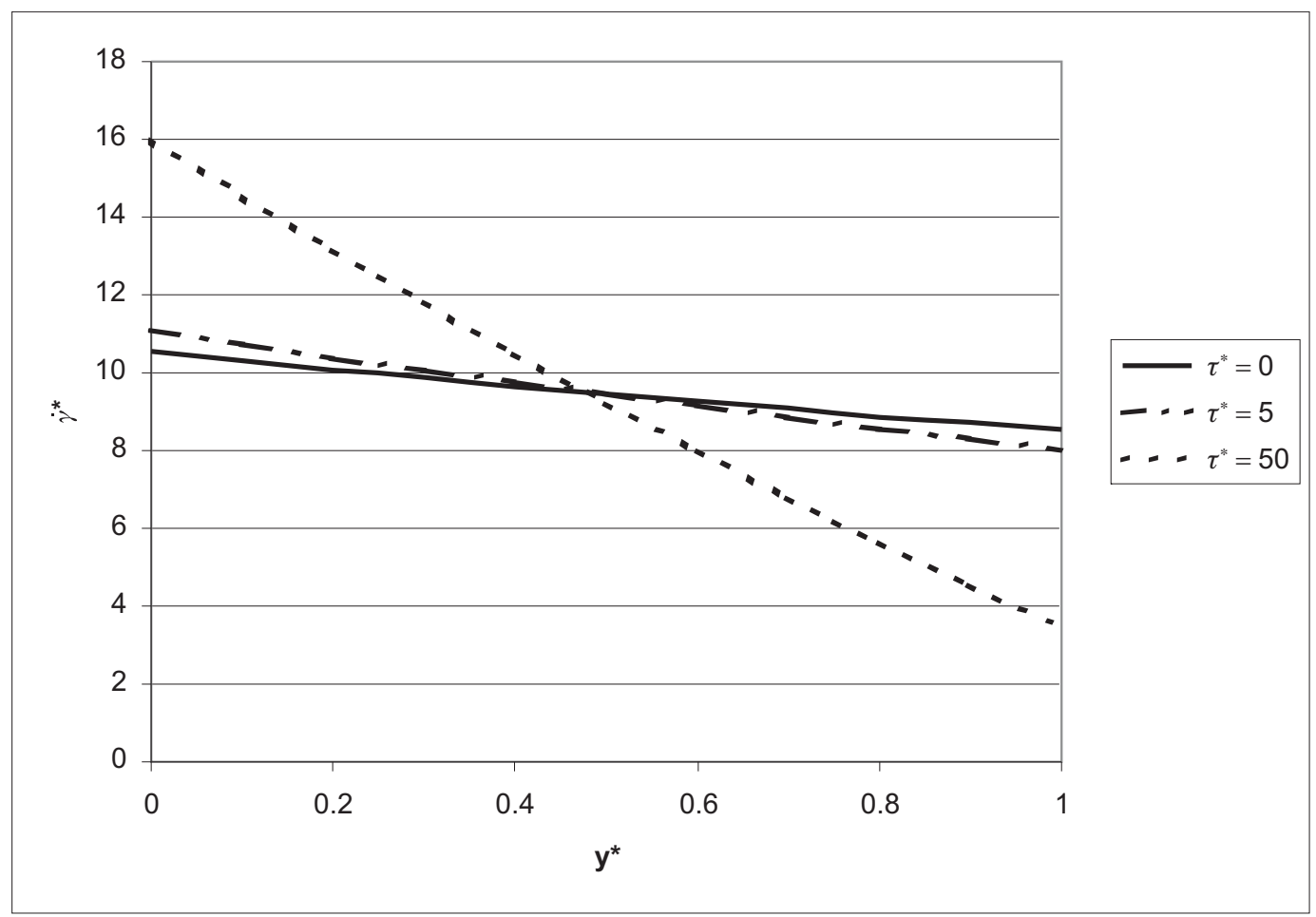

Fig. 5: Dependence $\dot{\gamma}^{*}$ on $y^{*}$ for $\kappa=0.9$ and selected values $\tau^{*}\left(\tau_{\mathrm{c}}^{*}=83.9\right)$ 
and after integration

$$
\frac{u_{\varphi}}{r}=\frac{\tau_{0}}{\mu_{\mathrm{p}}} \ln r-\frac{1}{2 \mu_{\mathrm{p}}} \frac{C_{1}}{r^{2}}+C_{2} .
$$

Using boundary conditions (7), we get, after some manipulation

$C_{1}=\frac{2 \omega \mu_{\mathrm{p}} R_{2}^{2} R_{1}^{2}}{R_{1}^{2}-R_{2}^{2}}-2 \tau_{0} \frac{R_{1}^{2} R_{2}^{2}}{R_{1}^{2}-R_{2}^{2}} \ln \frac{R_{1}}{R_{2}}$.

Combining Eqs. (3), (11) and (13) the equation for shear rate distribution can be obtained and the shear rate profiles shown in Figs. 4 and 5 can be depicted. From both figures it can be seen that $\dot{\gamma}^{*}$ distribution depends on $\kappa$ and the dimensionless parameter

$$
\tau^{*}=\frac{\tau_{0}}{\mu_{\mathrm{p}} \omega} .
$$

The distribution is more pronounced for greater $\tau^{*}$ and lower $\kappa$ values. However $\tau^{*}$ must be lower than critical at which shear stress value at the outer cylinder is equal to the yield stress $\tau_{2}=\tau_{0}$. At greater values of $\tau^{*}$, equation (10b) does not hold in the whole gap between the cylinders. The critical value $\tau_{\mathrm{c}}^{*}$ can be calculated from the equation

$$
\tau_{\mathrm{c}}^{*}=\frac{2 \kappa^{2}}{1-\kappa^{2}-2 \kappa^{2} \ln (1 / \kappa)}
$$

obtained from the condition $\tau_{r \varphi}=-\tau_{0}$ at $r=R_{2}$. The curves in Figs. 4 and 5 for $\tau^{*}=0$ are related to Newtonian fluids.

\section{Evaluation of rheological measurements}

The evaluation procedure is influenced by the radius to which the primary measured data (shear stress $\tau$ and the Newtonian shear rate $\dot{\gamma}_{N}$ ) are related.

\subsection{Power law fluids}

a) Data are related to inner radius $R_{1}$

Equation (4) at the inner cylinder surface takes the form

$$
\tau_{1}=K \dot{\gamma}_{1}^{n},
$$

where $\tau_{1}$ and $\dot{\gamma}_{1}$ are positive values of shear stress and shear rate at the inner cylinder surface. Using (9) we can obtain

$$
\dot{\gamma}_{1}=\frac{2 \omega}{n\left(1-\kappa^{2 / n}\right)} .
$$

However, the dependence of shear stress $\tau_{1}$ on the Newtonian shear rate $\dot{\gamma}_{1 N}$ at the surface of inner the cylinder is obtained from measurements. For this reason, equation (16) can be rewritten to the form

$\tau_{1}=K\left(\frac{\dot{\gamma}_{1}}{\dot{\gamma}_{1 N}}\right)^{n} \dot{\gamma}_{1 N}^{n}=K\left[\frac{1-\kappa^{2}}{n\left(1-\kappa^{2 / n}\right)}\right]^{n} \dot{\gamma}_{1 N}^{n}=K_{1} \dot{\gamma}_{1 N}^{n}$

where $\dot{\gamma}_{1 N}$ was expressed from (17) for $n=1$

$$
\dot{\gamma}_{1 N}=\frac{2 \omega}{1-\kappa^{2}}
$$

The dependences of the ratio $\dot{\gamma}_{1} / \dot{\gamma}_{1 N}$ on flow behavior index $n$ for cylinder ratio values $\kappa=0.5$ and 0.9 are depicted in Fig. 6. This figure shows that the values of this ratio (necessary for Newtonian shear rate correction) increase with decreasing flow behavior index $n$ and are significantly greater at $\kappa=0.5$ than at $\kappa=0.9$.

From (18) it can be seen that dependence $\tau_{1}$ on $\dot{\gamma}_{1 N}$ is a straight line with slope $n$ in logarithmic coordinates and the coefficient of consistency $K$ can be calculated by the equation

$$
K=K_{1}\left[\frac{n\left(1-\kappa^{2 / n}\right)}{1-\kappa^{2}}\right]^{n}
$$

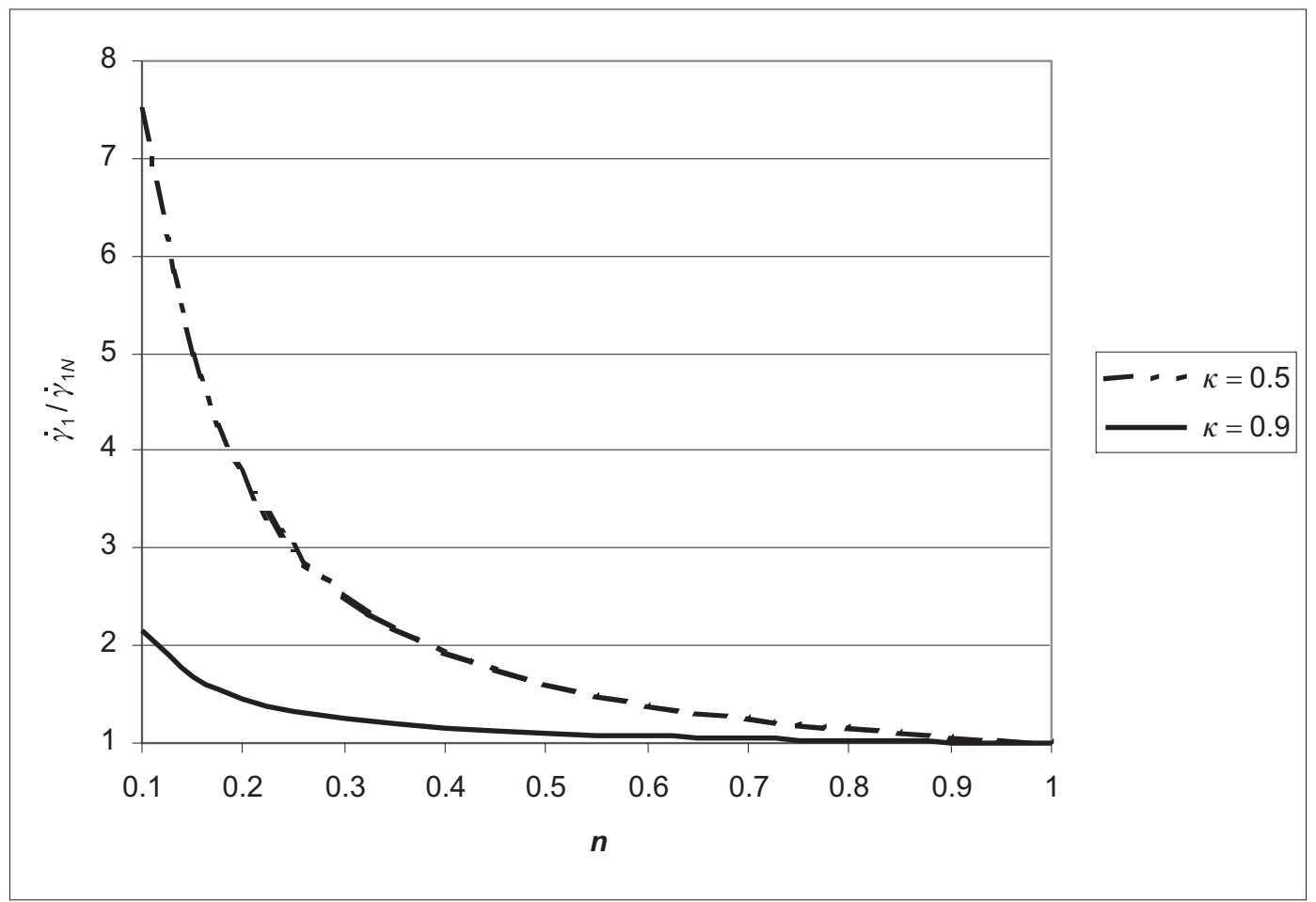

Fig. 6: Dependence of ratio $\dot{\gamma}_{1} / \dot{\gamma}_{1 N}$ on flow behavior index $n$ for selected $\kappa$ values 


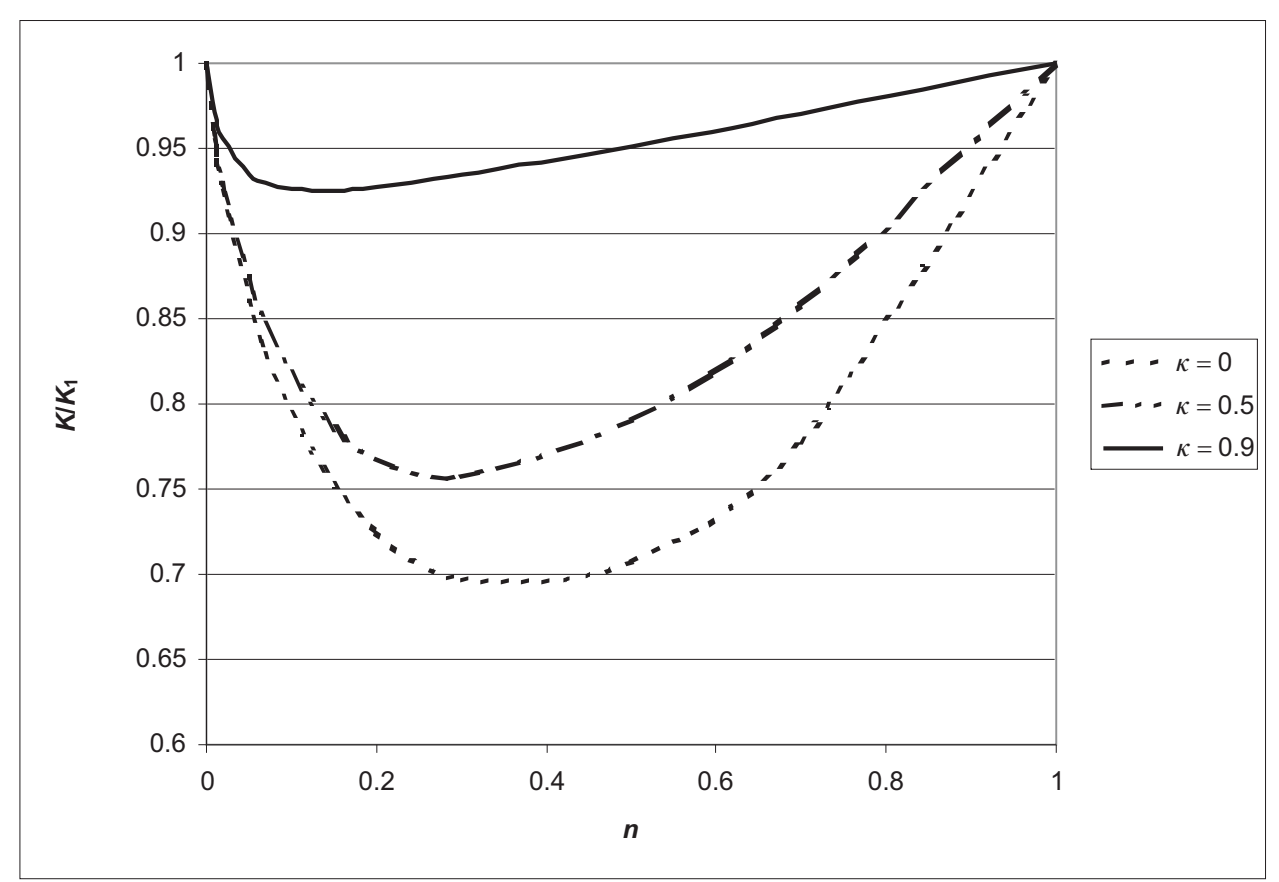

Fig. 7: Dependence of the ratio $K / K_{1}$ on flow behavior index $n$ for selected $\kappa$ values

The dependence of ratio $K / K_{1}$ on $n$ for several values $\kappa$ is shown in Fig. 7. From this figure it can be seen that it exhibits the minimum that is dependent on $\kappa$ (for $\kappa \rightarrow 0$, minimum $K / K_{1}=0.692$ at $\left.n=1 / \mathrm{e}=0.368\right)$.

b) Data related to mean radius $R_{\mathrm{m}}=\left(R_{1}+R_{2}\right) / 2$

Equation (4) written for the mean radius takes the form

$$
\tau_{\mathrm{m}}=K \dot{\gamma}_{\mathrm{m}}^{n}
$$

where $\tau_{\mathrm{m}}$ and $\dot{\gamma}_{\mathrm{m}}$ are positive values of shear stress and shear rate at the mean radius. Using (9) we can obtain

$\dot{\gamma}_{\mathrm{m}}=\frac{2 \omega}{n\left(1-\kappa^{2 / n}\right)}\left(\frac{R_{1}}{R_{\mathrm{m}}}\right)^{2 / n}=\frac{2 \omega}{n\left(1-\kappa^{2 / n}\right)}\left(\frac{2 \kappa}{1+\kappa}\right)^{2 / n}$.
However, the dependence of shear stress $\tau_{\mathrm{m}}$ on the Newtonian shear rate $\dot{\gamma}_{\mathrm{mN}}$ at the mean radius is obtained from measurements. For this reason, equation (21) can be rewritten to the form

$$
\tau_{\mathrm{m}}=K\left(\frac{\dot{\gamma}_{\mathrm{m}}}{\dot{\gamma}_{\mathrm{m} N}}\right)^{n} \dot{\gamma}_{\mathrm{m} N}^{n}=K_{\mathrm{m}} \dot{\gamma}_{\mathrm{m} N}^{n}
$$

where $\dot{\gamma}_{\mathrm{m} N}$ can be obtained from (22) for $n=1$

$$
\dot{\gamma}_{\mathrm{m} N}=\frac{2 \omega}{1-\kappa^{2}}\left(\frac{2 \kappa}{1+\kappa}\right)^{2} \text {. }
$$

Dependencies of $\dot{\gamma}_{\mathrm{m}} / \dot{\gamma}_{\mathrm{m} N}$ ratio on flow behavior index $n$ for cylinder ratio values $\kappa=0.5$ and 0.9 are depicted in Fig. 8 . This figure shows that the values of this ratio are less than 1

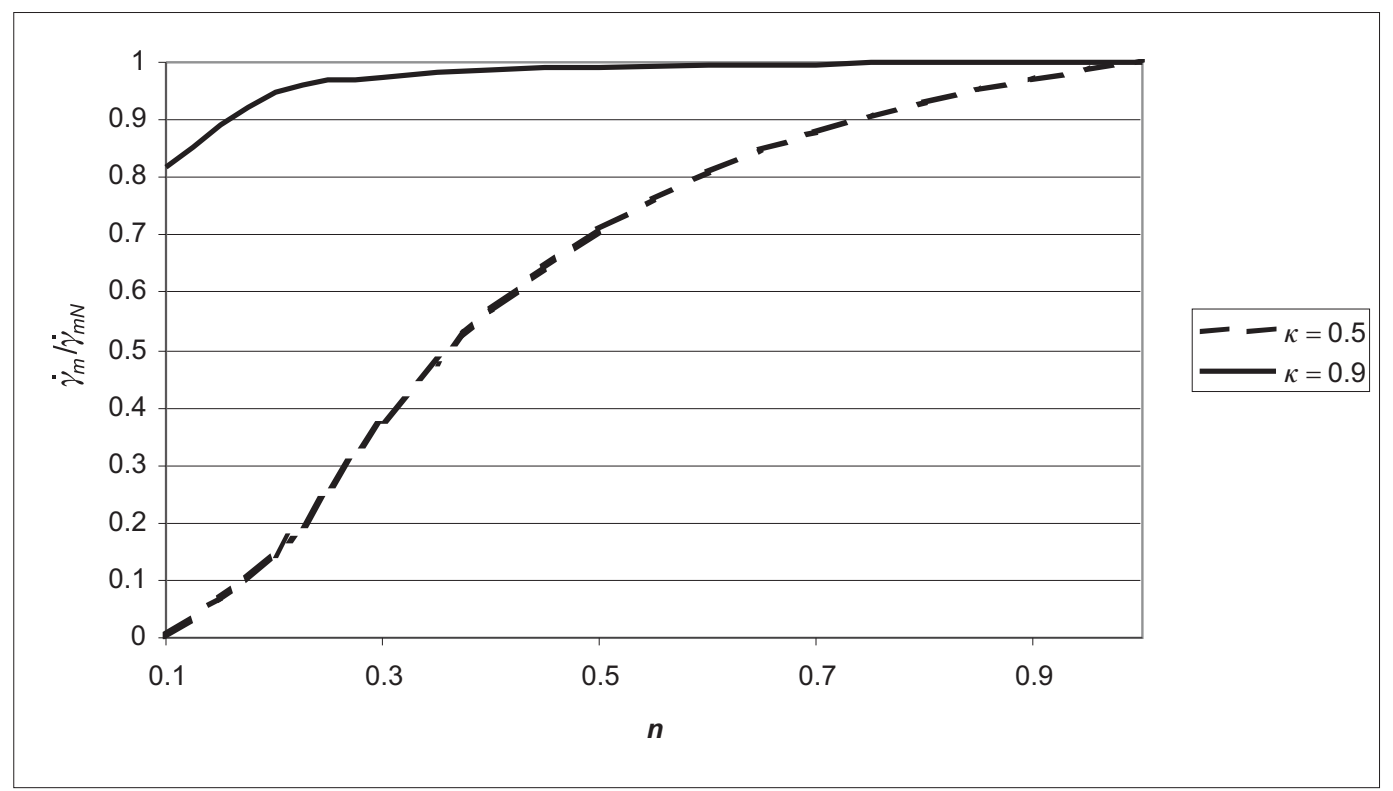

Fig. 8: Dependence of ratio $\dot{\gamma}_{\mathrm{m}} / \dot{\gamma}_{\mathrm{m} N}$ on flow behavior index $n$ for selected $\kappa$ values 


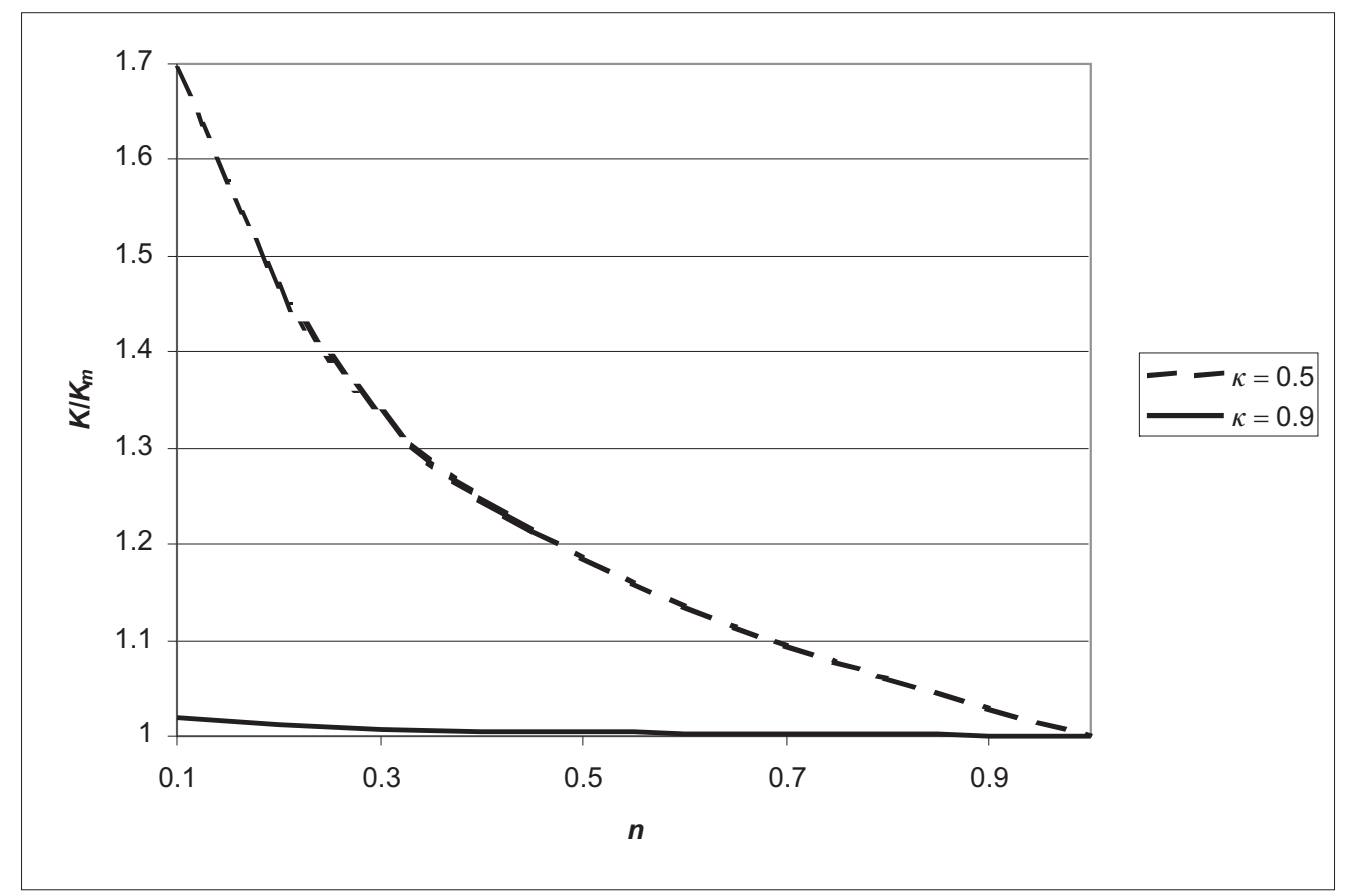

Fig. 9: Dependence of the ratio $K / K_{\mathrm{m}}$ on flow behavior index $n$ for selected $\kappa$ values

and decrease with decreasing flow behavior index $n$ and are significantly lower at $\kappa=0.5$ than at $\kappa=0.9$.

From (23) it can be seen that dependence $\tau_{\mathrm{m}}$ on $\dot{\gamma}_{\mathrm{m} N}$ is a straight line with slope $n$ in logarithmic coordinates and coefficient of consistency $K$ can be calculated by the equation

$$
K=K_{\mathrm{m}}\left(\frac{\dot{\gamma}_{\mathrm{m} N}}{\dot{\gamma}_{\mathrm{m}}}\right)^{n}
$$

the dependence of ratio $K / K_{\mathrm{m}}$ on $n$ for $\kappa=0.5$ and 0.9 is shown in Fig. 9. This figure shows that the ratio $K / K_{\mathrm{m}}$ is greater than 1 and it increases with decreasing $n$. c) Data related to the mean radius presented by Klein [2] $R_{\mathrm{K}}=R_{1} R_{2} \sqrt{\frac{2}{R_{1}^{2}+R_{2}^{2}}}$

Equation (4) written for this mean radius takes the form

$$
\tau_{\mathrm{K}}=K \dot{\gamma}_{\mathrm{K}}^{n} \text {, }
$$

where $\tau_{\mathrm{K}}$ and $\dot{\gamma}_{\mathrm{K}}$ are positive values of shear stress and shear rate at mean radius $R_{\mathrm{K}}$. Using (9) we can obtain

$\dot{\gamma}_{\mathrm{K}}=\frac{2 \omega}{n\left(1-\kappa^{2 / n}\right)}\left(\frac{R_{1}}{R_{\mathrm{K}}}\right)^{2 / n}=\frac{2 \omega}{n\left(1-\kappa^{2 / n}\right)}\left(\frac{1+\kappa^{2}}{2}\right)^{1 / n}$.

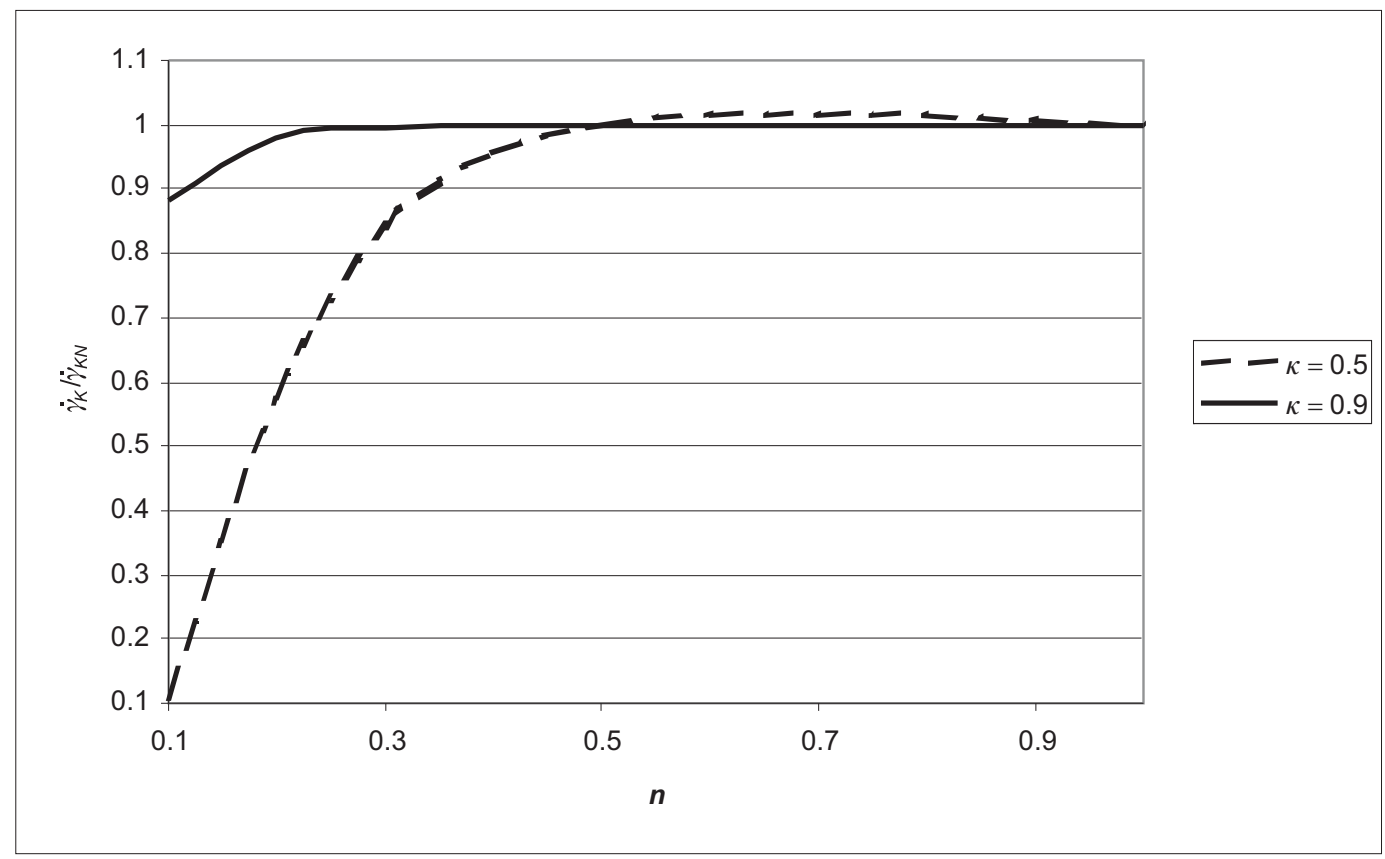

Fig. 10: Dependence of ratio $\dot{\gamma}_{\mathrm{K}} / \dot{\gamma}_{\mathrm{KN}}$ on flow behavior index $n$ for selected $\kappa$ values 


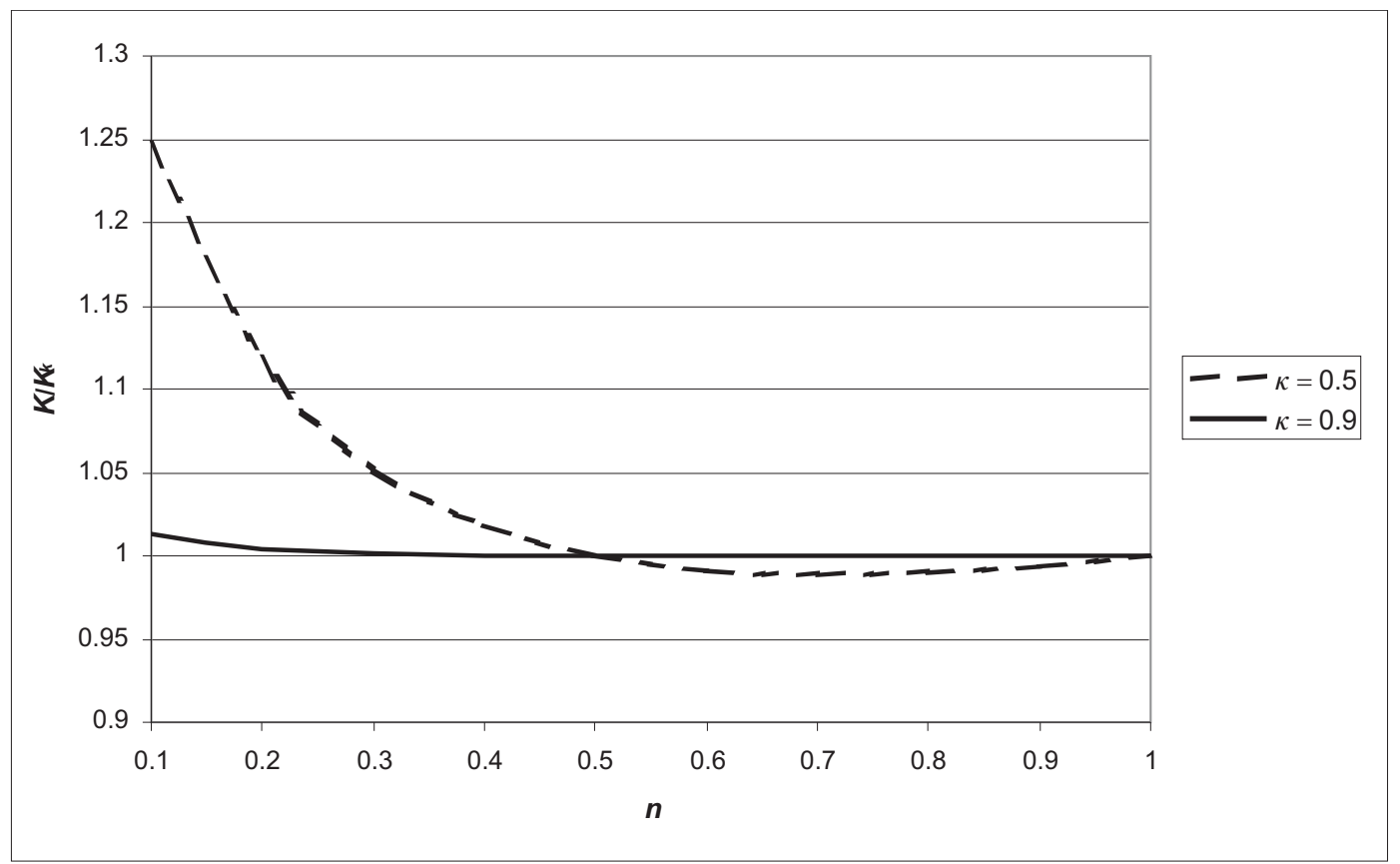

Fig. 11: Dependence of the ratio $K / K_{\mathrm{K}}$ on flow behavior index $n$ for selected $\kappa$ values

However, the dependence of shear stress $\tau_{\mathrm{K}}$ on the Newtonian shear rate $\dot{\gamma}_{\mathrm{KN}}$ at mean radius $R_{\mathrm{K}}$ is obtained from measurements. For this reason equation (26) can be rewritten to the form

$$
\tau_{\mathrm{K}}=K\left(\frac{\dot{\gamma}_{\mathrm{K}}}{\dot{\gamma}_{\mathrm{K} N}}\right)^{n} \dot{\gamma}_{\mathrm{K} N}^{n}=K_{\mathrm{K}} \dot{\gamma}_{\mathrm{K} N}^{n},
$$

where $\dot{\gamma}_{\mathrm{KN}}$ can be obtained from (27) for $n=1$

$$
\dot{\gamma}_{\mathrm{K} N}=\frac{1+\kappa^{2}}{1-\kappa^{2}} \omega .
$$

The dependencies of $\dot{\gamma}_{\mathrm{K}} / \dot{\gamma}_{\mathrm{K} N}$ ratio on flow behavior index $n$ for cylinder ratio values $\kappa=0.5$ and 0.9 are depicted in Fig. 10. This figure shows that the values of this ratio are ap- proximately 1 for average values of $n$ and rapidly decrease with decreasing flow behavior index $n$ at low $n$ values especially at $\kappa=0.5$.

From (28) it can be seen that dependence $\tau_{\mathrm{K}}$ on $\dot{\gamma}_{\mathrm{K} N}$ is a straight line with slope $n$ in logarithmic coordinates, and coefficient of consistency $K$ can be calculated by the equation

$$
K=K_{\mathrm{K}}\left(\frac{\dot{\gamma}_{\mathrm{K} N}}{\dot{\gamma}_{\mathrm{K}}}\right)^{n}
$$

the dependence of ratio $K / K_{\mathrm{K}}$ on $n$ for $\kappa=0.5$ and 0.9 is shown in Fig. 11 . This figure shows that ratio $K / K_{\mathrm{K}}$ is approximately 1 for usual values of $n$ and rapidly increases with decreasing flow behavior index $n$ at low $n$ values, especially at $\kappa=0.5$.

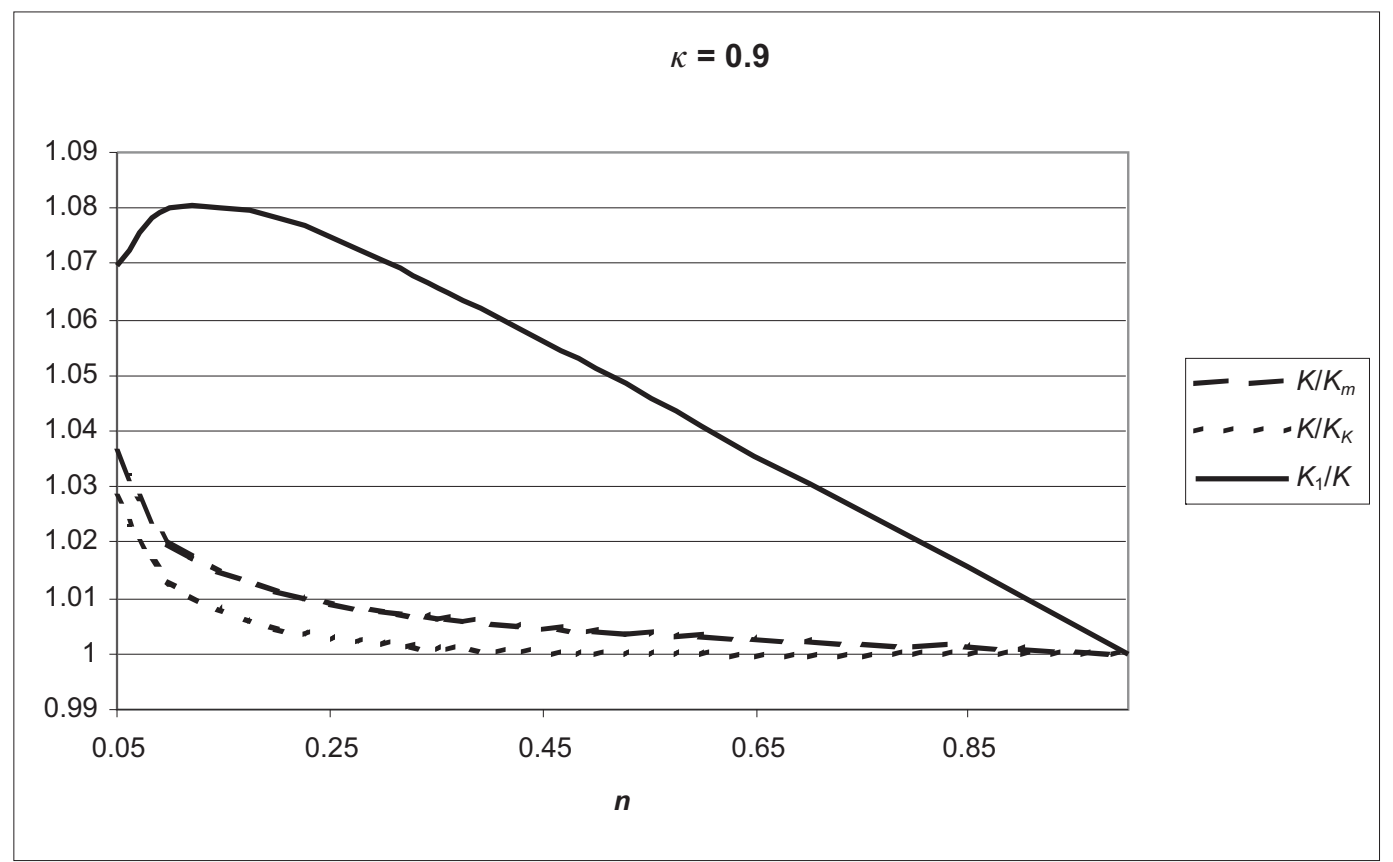

Fig. 12: Dependence of coefficient consistency ratios on flow index $n$ for $\kappa=0.9$ 


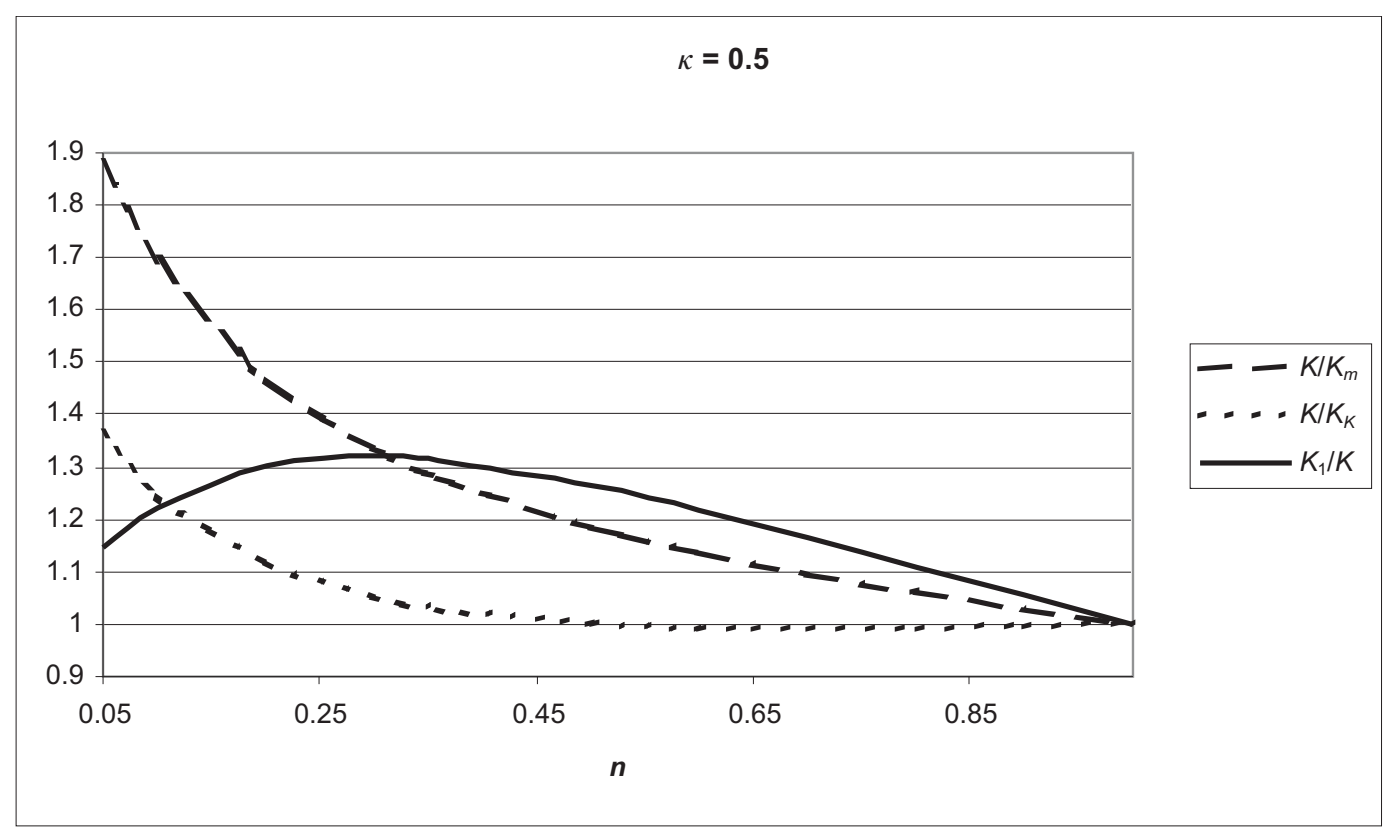

Fig. 13: Dependence of coefficient consistency ratios on flow index $n$ for $\kappa=0.5$

The ratios of the real and apparent consistency coefficients related to one of the radii mentioned above are compared in Figs. 12 and 13 for $\kappa=0.9$ and 0.5. Figs. 12 and 13 show that for $\kappa=0.9$ and for $\kappa=0.5$ and $n \geq 0.36$ no correction of $K$ is practically necessary (error is smaller than $3 \%$ ) when the data are related to the radius defined according to Klein.

\subsection{Bingham plastics}

The dependences of the ratio of the shear rate to the Newtonian shear rate (related to the reference radii) on $\tau^{*}$ for $\kappa=0.5$ and 0.9 are shown in Figs. 14 and 15. These figures shows that the values of shear rate related to radii $R_{1}$ and $R_{\mathrm{m}}$ are greater than the Newtonian values and the shear rate values related to radius $R_{\mathrm{K}}$ are smaller than the Newtonian values.

Inserting (13) into (2) we derive that the dependence of shear stress on Newtonian shear rate values (related to reference radii) can be expressed in the form

$$
\tau=\tau_{0 \text { ref }}+\mu_{\mathrm{p}} \dot{\gamma}_{N} \text {. }
$$

The ratio $\tau_{0 \text { ref }} / \tau_{0}$ depends on $\kappa$ and the reference radius to which the measured data are related and the following formulas can be derived:

a) Data related to inner radius $R_{1}$

$$
\frac{\tau_{01}}{\tau_{0}}=\frac{2 \ln (1 / \kappa)}{1-\kappa^{2}}
$$

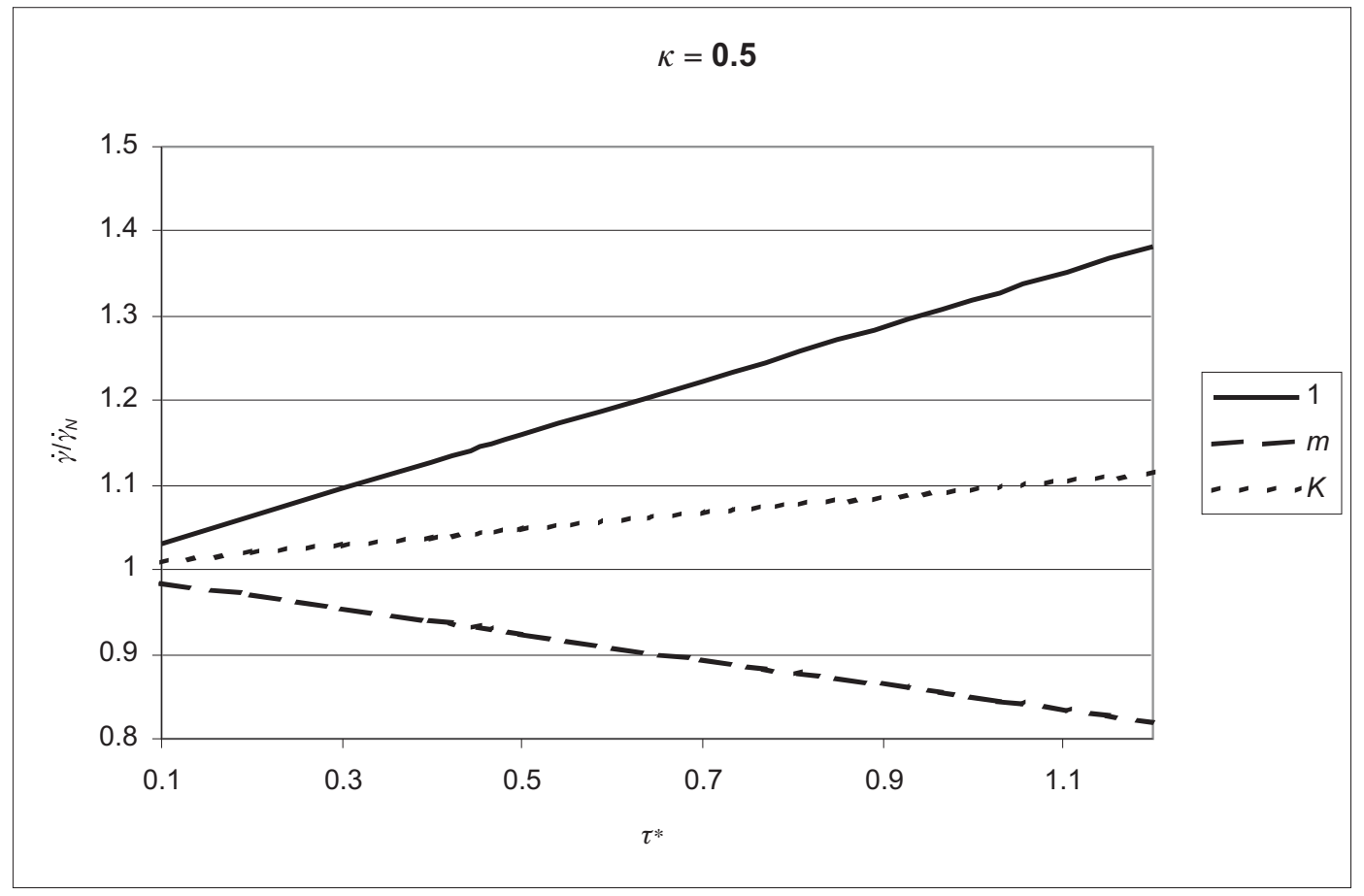

Fig. 14: Dependences of shear rate to Newtonian shear rate ratio (related to reference radii) on $\tau^{*}$ for $\kappa=0.5$ 


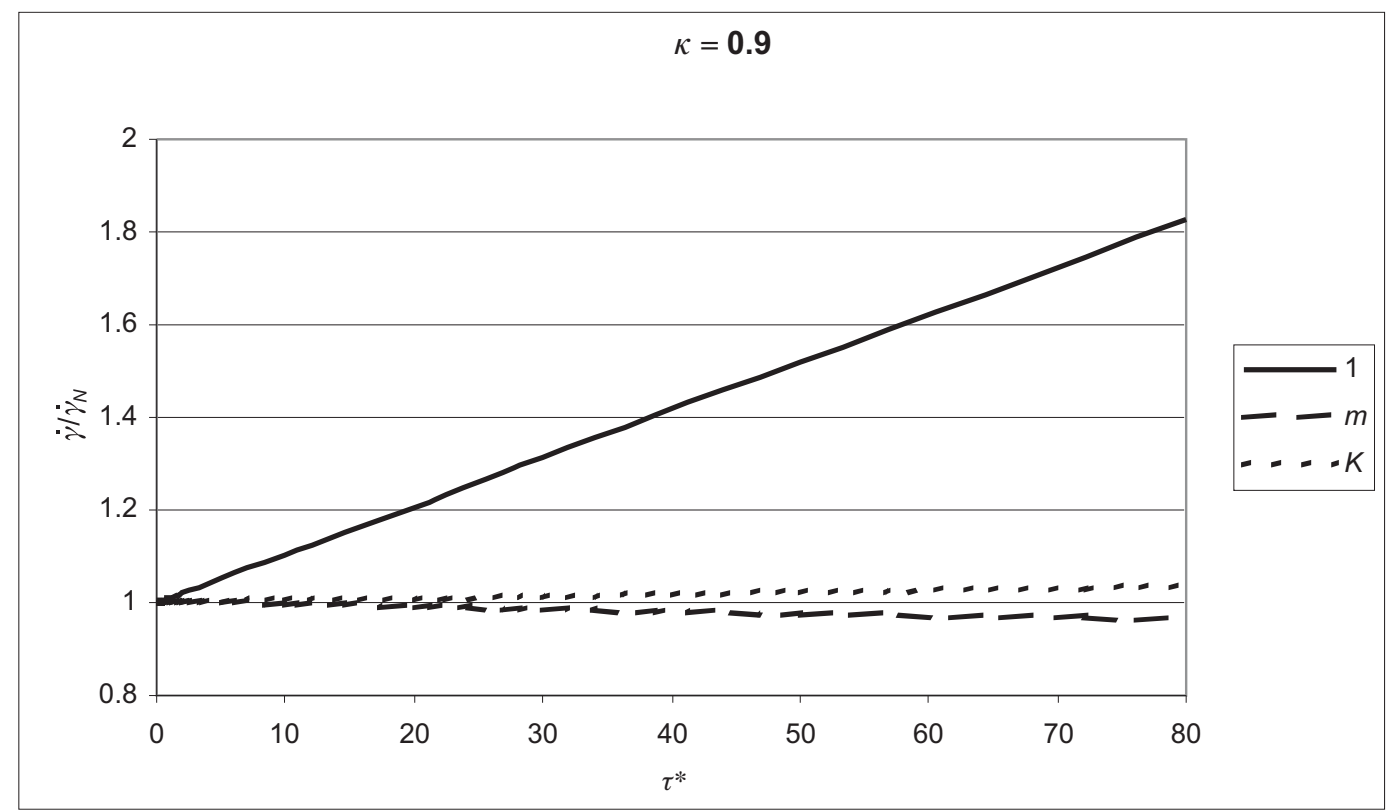

Fig. 15: Dependences of shear rate to Newtonian shear rate ratio (related to reference radii) on $\tau^{*}$ for $\kappa=0.9$

b) Data related to mean radius $R_{\mathrm{m}}=\left(R_{1}+R_{2}\right) / 2$

$$
\frac{\tau_{0 \mathrm{~m}}}{\tau_{0}}=\frac{8 \kappa^{2} \ln (1 / \kappa)}{\left(1-\kappa^{2}\right)(1+\kappa)^{2}}
$$

c) Data related to the mean radius presented by Klein [2]

$$
\begin{aligned}
R_{\mathrm{K}}=R_{1} R_{2} \sqrt{\frac{2}{R_{1}^{2}+R_{2}^{2}}} \\
\frac{\tau_{0 \mathrm{~K}}}{\tau_{0}}=\frac{1+\kappa^{2}}{1-\kappa^{2}} \ln \frac{1}{\kappa} .
\end{aligned}
$$

The dependences of ratio $\tau_{0 \text { ref }} / \tau_{0}$ on $\kappa$ for different reference radii are depicted in Fig.16. This figure shows that practically no correction is necessary for $\kappa \geq 0.74$ when the data are related to the radius defined according to Klein (the error is smaller than $3 \%$ ).

\section{Conclusion}

The following procedure can be recommended for determining the rheological model parameters:

1) Comparing the values of $\omega$ and $\dot{\gamma}_{N}$ reported by the manufacturer of the viscometer, we determine reference radius to which measured data are related (Eq.(19), (24), (29)).

2) From the measured shear stress and Newtonian shear rate values the values of $K_{\text {ref }}$ and $n$ are obtained for power-law

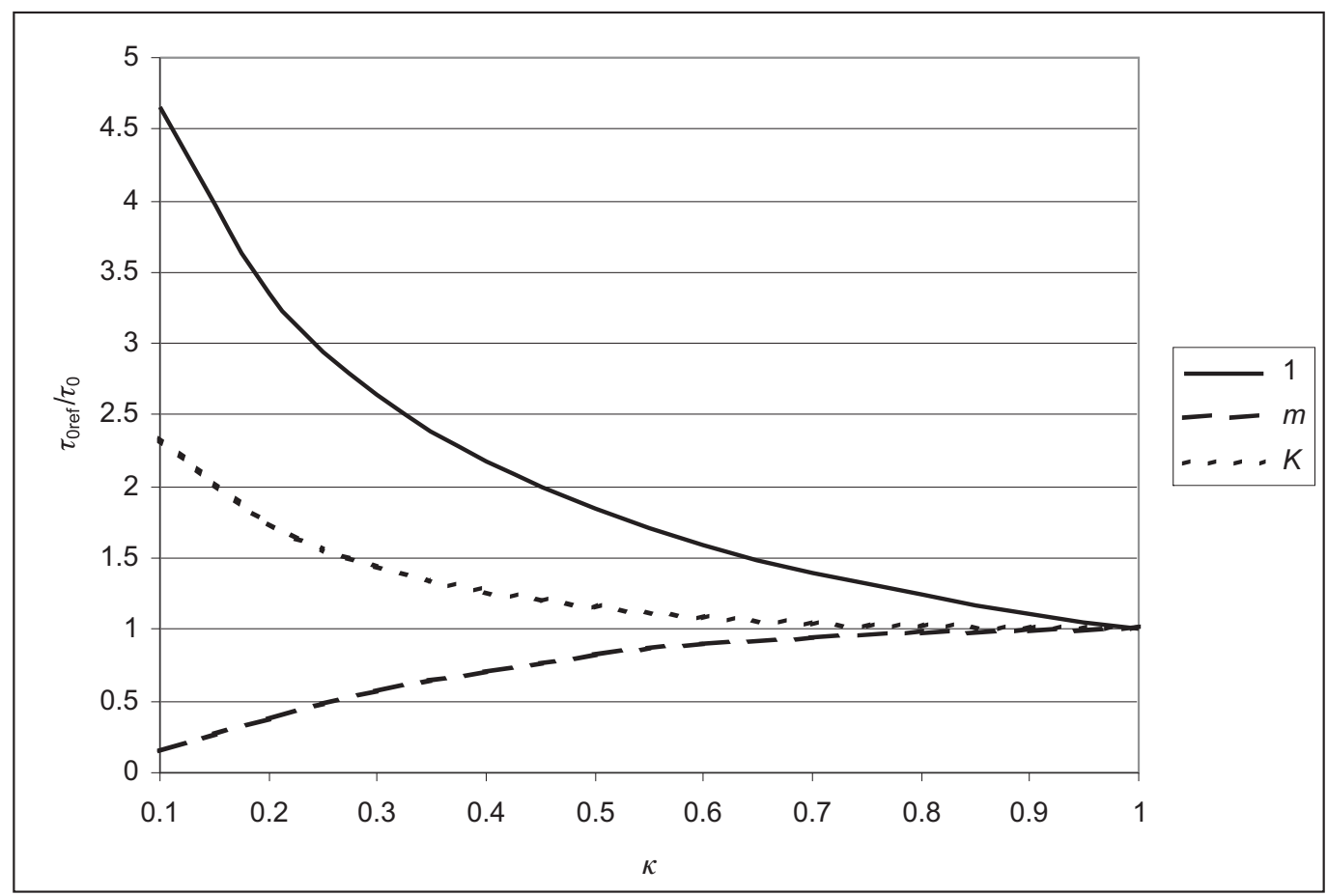

Fig. 16: Dependences of ratio $\tau_{0 \text { ref }} / \tau_{0}$ on $\kappa$ for different reference radii 
fluids (Eq.(18), (23), (28)) or $\tau_{\text {Oref }}$ and $\mu_{\mathrm{p}}$ are obtained for Bingham plastics (Eq.(31)).

3) Values of the rheological model parameters, i.e., consistency coefficient $K$ (Eq.(20), (25), (30)) or yield stress $\tau_{0}$ (Eq.(32), (33), (34)) must be determined.

\section{List of symbols}

$K \quad$ coefficient of consistency

$L \quad$ length of cylinder

$n$ flow index

$r$ radial coordinate

$R_{1} \quad$ inner rotating cylinder radius

$R_{2} \quad$ outer stationary cylinder radius

$u \quad$ velocity

$\dot{\gamma}$ shear rate

$\varphi \quad$ tangential coordinate

$\kappa \quad R_{1} / R_{2}$ ratio

$\mu_{\mathrm{p}} \quad$ plastic viscosity

$\omega \quad$ angular velocity $=R_{1} / R_{2}$ $\tau \quad$ shear stress

$\tau_{0} \quad$ yield stress

\section{References}

[1] Middleman, S.: The Flow of High Polymers. New York: Interscience Publishers, 1968.

[2] Klein, G.: Basic Principles of Rheology and the Application of Rheological Measurement Methods for Evaluating Ceramic Suspensions. In: Ceramic Forum International Yearbook 2005 (Edited by H. Reh). Baden-Baden: Göller Verlag, 2004, p. 31-42.

Prof. Ing. František Rieger, DrSc. phone: +420224352548

email: frantisek.rieger@fs.cvut.cz

Czech Technical University in Prague

Faculty of Mechanical Engineering

Technická 4

16607 Praha 6, Czech Republic 